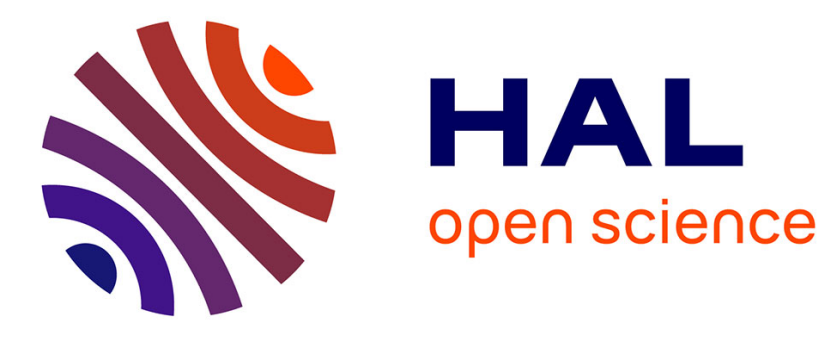

\title{
Resist trimming etch process control using dynamic scatterometry
}

Mohamed El Kodadi, Sébastien Soulan, Maxime Besacier, Patrick Schiavone

\section{To cite this version:}

Mohamed El Kodadi, Sébastien Soulan, Maxime Besacier, Patrick Schiavone. Resist trimming etch process control using dynamic scatterometry. Microelectronic Engineering, 2009, 86 (4-6), pp.10401042. 10.1016/j.mee.2008.12.036 . hal-00374855

\section{HAL Id: hal-00374855 https://hal.science/hal-00374855}

Submitted on 10 Apr 2009

HAL is a multi-disciplinary open access archive for the deposit and dissemination of scientific research documents, whether they are published or not. The documents may come from teaching and research institutions in France or abroad, or from public or private research centers.
L'archive ouverte pluridisciplinaire HAL, est destinée au dépôt et à la diffusion de documents scientifiques de niveau recherche, publiés ou non, émanant des établissements d'enseignement et de recherche français ou étrangers, des laboratoires publics ou privés. 


\title{
Resist trimming etch process control using dynamic scatterometry
}

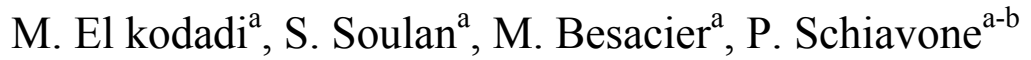 \\ a Laboratoire des Technologies de la Microélectronique CNRS, 17 rue des Martyrs, 38054 \\ Grenoble,France \\ b UMI 2958 Georgia Tech-CNRS, Georgia Institute of Technology, 777 Atlantic Dr., Atlanta \\ (GA) 30332, USA
}

Elsevier use only: Received date here; revised date here; accepted date here

\begin{abstract}
Dynamic scatterometry is an optical metrology technique designed for the in-situ real time process control in the production of microelectronics devices. This technique has many advantages; however the main limitation in the real time context comes from the short data acquisition time for the needed wavelength range. We present here the different tools to perform dynamic scatterometry, such as specific software and hardware tools. We show how these tools can successfully be used to monitor the resist etch process in an industrial etch chamber from Applied Materials equipped with an in-situ ellipsometer. Results from determining $\mathrm{CD}$ and thickness shows excellent agreement between the scatterometry measurements and measurements made with a 3D AFM.
\end{abstract}

(C) 2008 Elsevier Science. All rights reserved

Keywords: Scatterometry, Real time, Resist trimming, In situ metrology, Ellipsometry

\section{Introduction}

Various techniques have been proposed and implemented for measuring very small dimensions with a good accuracy. Atomic Force Microscopy (AFM) and CD Scanning Electron Microscopy CDSEM can deliver direct morphological images of very small features. However, they are very expensive and potentially destructive, thus not suitable for real time monitoring, either because they are inherently slow or bulky equipments makes impossible.

Indeed, in-line process control in microelectronic manufacturing requires real-time and non-invasive monitoring techniques. Optical measurements are among the few solutions able to meet these requirements. Scatterometry belongs to the optical strategies, it uses the analysis of the signature of the light scattered by a periodic structure to deduce the shape of a feature (for example using spectroscopic ellipsometry). It has been shown to be versatile and

a Corresponding author. Tel.: +33-43-878-0397; fax+33-43-8789292;e-mail: mohamed.el-kodadi@cea.fr applicable to in-situ real time process control in the production of microelectronics devices. The inverse problem which allows finding the grating shape using an experimental signature acquisition is generally solved by the method of library searching [1]. The so called "direct problem" of computing the optical response of a defined shape is dealt with using electromagnetic solvers, typically based on modal methods. This technique has non- destructive, noninvasive, and can be used for in situ real time monitoring

Soulan et al [2] have developed specific software and hardware tools to perform dynamic scatterometry, using the in situ spectroscopic multiwavelength ellipsometer.

This paper present the first results of the application of dynamic scatterometry for the monitoring of a $\mathrm{HBr}$ and $\mathrm{O}_{2}$, resist trimming process.

The etching process chosen for this first validation consists in trimming $500 \mathrm{~nm}$ dense resist features. The measurement is made in real time, using an 
ellipsometer capable of real time acquisition of 16 wavelengths simultaneously.

\section{Experimental details}

The resist trimming experiments are performed with a commercial high density plasma source DPS CENTURA 5200B from Applied Materials, sketches of this Decoupled Plasma Source (DPS) silicon etch system has been previously presented elsewhere [3],[4]. The DPS is a high density source where both the source antenna and the bottom electrode are powered at a slightly different frequency respectively $12.56 \mathrm{MHz}$ and $13.56 \mathrm{MHz}$; the source power controls the ion density, while the bottom power controls the energy of ions striking the wafer surface. The etch gases used in this study are $\mathrm{HBr}$ and $\mathrm{O} 2$. The in-situ spectroscopic ellipsometer is a Jobin-Yvon multiwavelength MWL-16, and the incident angle is constant at $72.32^{\circ}$, the beam size is an elliptical region $2 \mathrm{~mm} \times 6 \mathrm{~mm}$. The $500 \mathrm{~nm}$ equal line to space range resist patterns are obtained from a M78Y resist from JSR, exposed to a 248nm using a ASML 300 exposure tool.

The ellipsometry measures the change in the amplitude $\Psi$, and phase $\Delta$, between the $p$ (parallel) and the $s$ (perpendicular) components of polarized light upon reflection from a surface [5]. $\Psi$ and $\Delta$ are given in terms of the complex reflection coefficients $r_{p}, r_{s}$ :

$$
\begin{aligned}
& \frac{r_{p}}{r_{s}}=\rho=\tan \Psi e^{i \Delta} \\
& I_{s}=\sin 2 \Psi \sin \Delta \\
& I_{c}=\sin 2 \Psi \cos \Delta
\end{aligned}
$$

During etching, the multi-wavelength ellipsometer can monitor the real time data (Is, Ic) at sixteen photon energies between $1.526 \mathrm{ev}$ and 4.834 ev every $0.63 \mathrm{~s}$, these data are accorded to $\Psi$ and $\Delta$ by equations (1.2) and (1.3).

A bench of scatterometry is represented in Fig1, we typically operate the ellipsometer in a configuration such that $P-M= \pm 45^{\circ}, M=0 \pm 90^{\circ}$, and $A= \pm 45^{\circ}$, where $A, M$, and $P$ are the angles referenced to the plane of incidence of the analyzer, modulator, and polarizer respectively.
The scatterometric analysis can be defined in two steps. First, the theoretical scatter signatures were computed using a rigorous electromagnetic solver based on the Modal Method by Fourier Expansion (MMFE) detailed by Li et al [6]. This is known as the direct problem. This technique uses a modal decomposition of Maxwell's equations with appropriate boundary conditions at each interface of the geometric structure to solve the electric field in each region of the sample, and compute the ellipsometric quantities.

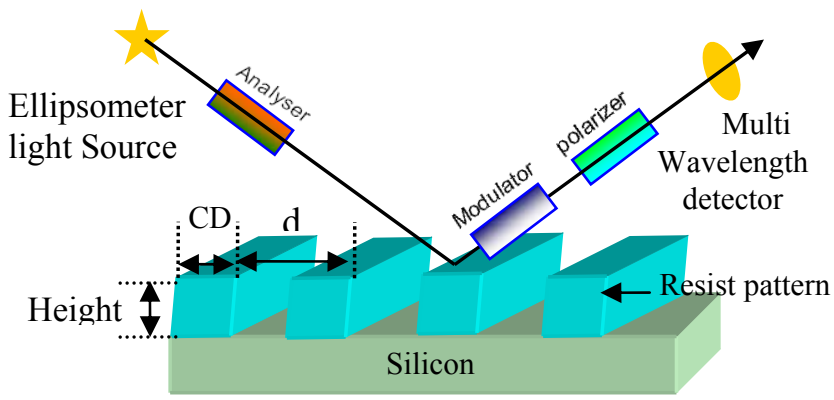

Fig1. Schematic drawing of the experimental Setup.

The second step, the inverse problem, consists in determining the shape of the lines of the periodic structure from an experimental signature acquisition. To solve this problem, different classes of optimisation algorithms have been used ( evolutionary, simplex, etc. ) [7], but the method of library searching [1] is the most commonly used in the industry. Moreover, this method is real time compliant as the solving time is perfectly predictable.

A signature library is built-up with direct problem using different combinations of profile parameters (CD, height, sidewall angle, etc.). The experimental signature is compared with the signatures of the library, in order to obtain the best match. Although, generating the library requires a lot of computation time, and require high performance processor, solving the inverse problem can be fast because pattern matching is reduced here to a simple numerical database search. Unfortunately, this technique becomes difficult and looses accuracy when scatterometry data are limited in wavelength resolution. Indeed, due to the acquisition speed requirement imposed by real time monitoring, most experimental set-ups put a limitation to a much smaller number of wavelengths than in the static 
case. Consequently, in the case of real time analysis, the standard library method fails because several database patterns can match the experimental data, not to mention local minima. Since it is too much time consuming to solve the inverse problem for each time step, our idea is based on the following: a full resolution signature is measured at $\mathrm{t}=0$ before processing and subsequent partial signatures with much lower wavelength resolution are used to refine the library search using a time-based Tikhonov regularization procedure[2],[8]. In this method, the best match to an element in the library is selected by using $\mathrm{k}$ nearest neighbors $(\mathrm{k}-\mathrm{NN})$, followed by an adjustment in time in order to appropriately balance the proximity to the measured data and the smoothness of shape variation over time. Figure 2 illustrates this concept

The regularization has to be made into a time sliding window in order to limit computing resource.

This technique has been implemented as a dedicated $\mathrm{C}++$ software architecture. We used a GPU (Graphic Processing Unit) as a numerical database accelerator for the library-based inverse problem. Due to the inherent vector architecture of the GPU [9], the entire library search operation can be made in only a few clock cycles. A full description of this method can be found elsewhere [2]

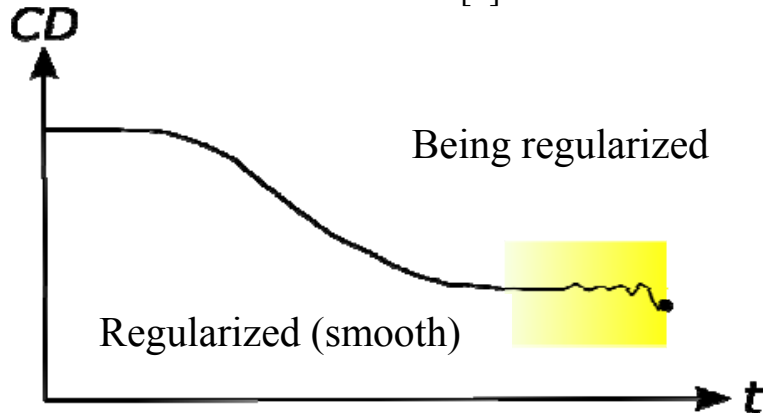

Fig2. Real time reconstruction takes place within a sliding time window (yellow in the graph)

\section{Results and discussion}

In this study the first experimental validation of the application of dynamic scatterometry to plasma resist trimming process, are disclosed to show the validity of our algorithms. The etching process of interest for this validation is the resist trimming $500 \mathrm{~nm}$ dense features of $248 \mathrm{~nm}$ resist.
Resist trimming is an essential step for the manufacturing of sub-20 nm gate length transistors. This step consists in consuming laterally and vertically resists features to reduce their size. It generally uses oxygen mixed with $\mathrm{HBr}$ or $\mathrm{Cl}_{2}$, in our case the gas mixture used in this study is $\mathrm{HBr} / \mathrm{O}_{2}$ $(70: 30 \mathrm{sccm})$. The plasma source was operated at $300 \mathrm{~W}$ source power; the pressure in the reactor was 4 mTorr with $0 \mathrm{~W}$ bias power. For the inverse problem, the features are considered as rectangular shaped and the only parameters to be determined in this study are the $\mathrm{CD}$, and the grating height. The period $\mathrm{d}$ is fixed to $1 \mu \mathrm{m}$. The grating is placed on top of thin films with a silicom substrate (Fig1). It has been demonstrated that in these experimental conditions the isotropy of the etching is obtained (Fig3).

We checked that materials optical properties are not modified with this process. Indeed potential material modifications during the etching process remain a big challenge for dynamic scatterometry. For validation purposes, the process has been interrupted at several different times and the trimmed feature profiles have been measured using an atomicforce microscope X3D from Veeco Instruments.

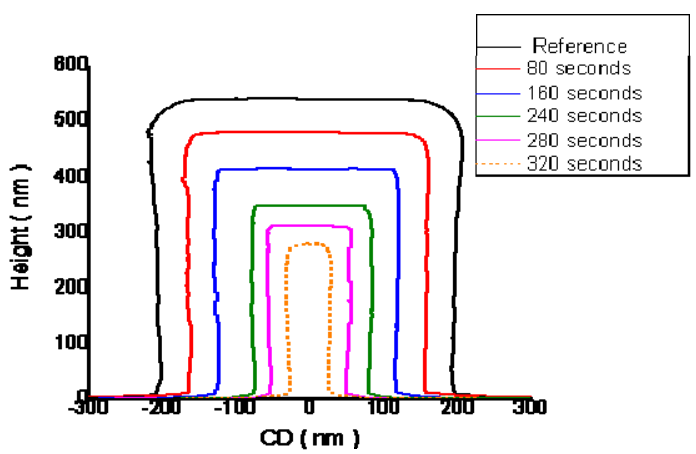

Fig3. AFM section image of the $500 \mathrm{~nm}$ features of $248 \mathrm{~nm}$ resist at different times.

AFM results in 3 dimensional images of the surface, enabling to measure accurately vertical lines (CD and height), the spaces between the lines as well as the sidewall roughness of the feature [10]. We measured with AFM the average profiles for 20 lines of scan to have an measurement of the resulting CD and height (Fig3). The AFM measures show that the most important parameters which vary during etching process are the $\mathrm{CD}$ and Height, and confirm the isotropy of the etch process. Scattering results were 
measured in real time using the multi-wavelength ellipsometer.

A comparison between dynamics scatterometry profile shape (CD, height) and 3D AFM measurements made in the same condition and at the different time step is shown in fig4 and fig5.

We can clearly see that the reconstructed profile matches well the measurement made with AFM, both for the $\mathrm{CD}$ and height. This preliminary study showed that the dynamic scatterometry for shape reconstruction can provide reliable results and that it shows a great potential to be used as a real time monitoring technique for etch process control.

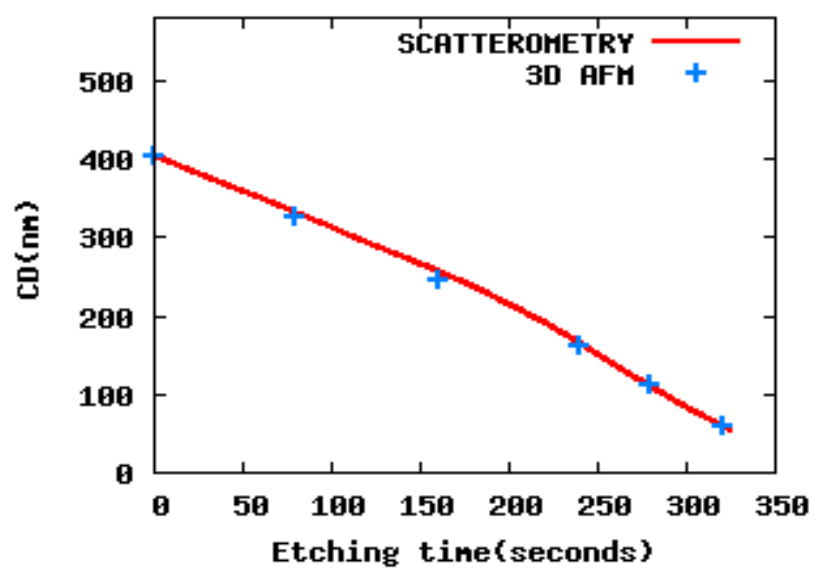

Fig4. Real time measurement results $C D$ of the resist feature over the processing time. 3D AFM data are shown for reference

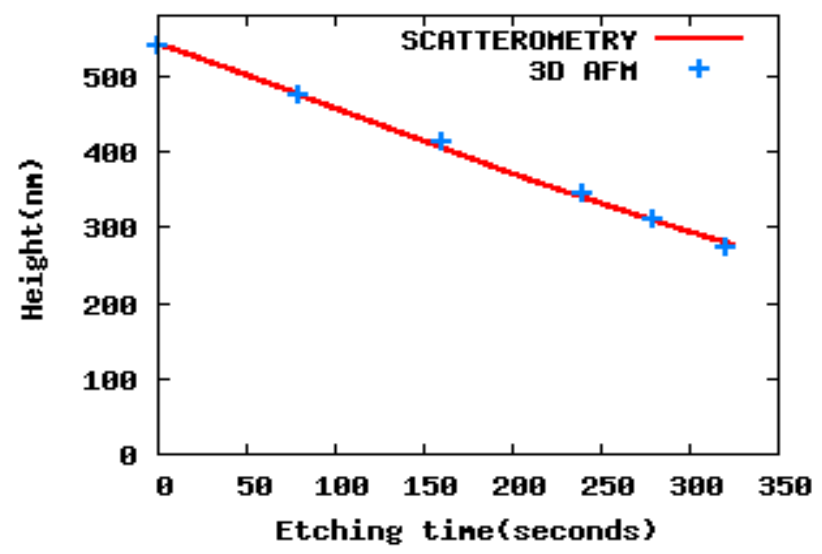

Fig5. Real time measurement results Height of the resist feature over the processing time. $3 D$ AFM data are shown for reference

\section{Conclusion and perspectives}

We have presented a very efficient in situ metrology method namely dynamic scatterometry used in real time during a resist trimming process. By using in situ spectroscopic ellipsometry, we have applied and validated this technique to the particular case of $\mathrm{HBr} / \mathrm{O} 2$ resist trimming process. However; an important effort remains to do mainly in two directions.

First, the parameters of the regularization used in our inverse problem optimization can affect the results in a significant way. It must be adapted to each process under test. More work has to be done in order to determine precisely the best regularization parameters for each process.

Second, experimental validations are still necessary in order to enhance the confidence and assess the range of validity of this extremely promising technique. We are studying the application to more complex plasma etching processes. This opens up other challenges such as considering material modifications during the etching process.

\section{References}

[1] X. Niu, N. Jakatdar, J. Bao, and C. J. Spanos, IEEE transactions on semiconductor manufacturing, VOL. 14, NO. 2 , MAY 2001

[2] S. Soulan, M. Besacier, P. Schiavone; Proceedings of the SPIE conference on Modelling Aspects in Optical Metrology, vol. 6617, 2007.

[3] G.Cunge, R. L. Inglebert, O. Joubert, L.Vallier, and N.Sadeghi, J.Vac Sci. Technol. B 20, 2137 (2002).

[4] Helen L. Maynard, Nacer Layadi, and John Tseng-Chung Lee J. Vac. Sci. Technol. B 15(1), Jan/Feb 1997.

[5] R. M Azzam and N.M Bashara, Ellipsometry and Polarized light, North-Holland (1977).

[6] Lifeng Li. New formulation of the Fourier modal method for crossed surface relief gratings. J. Opt. Soc. Am. A, 14(10), Oct 1997.

[7] C.J. Raymond et al, Proceeding of the SPIE vol. 5375, pp. 564575 (2004).

[8] A. Tikhonov and V. Arsenin, solution of Ill-posed Problems, (Wiley, New york, 1977).

[9] B. Busstos, et al, A Graphics Hardware Accelerated algorithm for Nearest Neighbor Search, lecture notes in computer science, 2006. Springer, Vol. 3994/2006, pp. 196-199.

[10] J.Foucher, A. L. Fabre, P. Gautier Proc. SPIE 6152, 2006. 